\title{
Método espectroscópico para determinação de cumarina em xarope de Mikania glomerata Sprengel
}

\author{
Cleinils R. da Silva, Víctor S. Gomes, Irene C. Kulkamp, Luiz A. Kanis*
}

Grupo de Pesquisa em Tecnologia Farmacêutica, Curso de Farmácia, Universidade do Sul de Santa Catarina, Av José Acácio Moreira 787, Caixa Postal 307, 88704-900 Tubarão-SC, Brasil

\begin{abstract}
RESUMO: Este trabalho teve como objetivo desenvolver uma metodologia analítica por espectroscopia UV, para doseamento de cumarina em xaropes de Mikania glomerata. A técnica foi baseada na extração da cumarina utilizando solventes como o clorofórmio e hexano. Após a seleção do solvente, o comprimento de onda foi definido através da sobreposição dos espectros da cumarina, metil parabeno, diluição do xarope e solução extrativa do xarope. Foram preparadas curvas analíticas de cinco soluções de cumarina com concentração variando de 0,002 a 0,03 $\mathrm{mg} / \mathrm{mL}$. Para análise da exatidão do método, foram preparados três lotes de xarope de Mikania glomerata Sprengel e o teor de cumarina determinado pela técnica espectrofotométrica foi comparado a técnica por cromatografia líquida de alta eficiência. O solvente selecionado para extração foi o clorofórmio, o comprimento de onda $320 \mathrm{~nm}$. A curva analítica apresentou $\mathrm{R}^{2}$ de 0,99978, demonstrando linearidade. A comparação estatística do doseamento da cumarina pela técnica espectrofotométrica estudada com a técnica cromatográfica desenvolvida por Celeghini et al. (2001) demonstrou não existir diferenças significativas, indicativo de exatidão da técnica.
\end{abstract}

Unitermos: Mikania glomerata, Asteraceae, cumarina, espectrofotômetro, metil parabeno, xarope.

\begin{abstract}
Ultraviolet spectroscopy method for coumarin quantification in Mikania glomerata Sprengel syrup". A spectrophotometric procedure for coumarin determination in Mikania glomerata Sprengel (guaco) syrup is described in this work. Due to the high number of constituents in guaco syrups, the coumarin was extracted with apolar extractors (chloroform and hexane), in which chloroform was selected, because of its higher capacity of extraction. After the solvent choice, the wavelength at $320 \mathrm{~nm}$, region where there is the lower interference of syrup constituents, was selected. The calibration curve showed linearity, $\mathrm{R}^{2}$ of 0.99978 . The spectrophotometric assay of coumarin in three samples of Mikania glomerata Sprengel syrup showed accuracy compared with the HPLC method. The results presented suggest that the spectrophotometric method may be useful for the quantitative analysis of coumarin in Mikania glomerata Sprengel syrup.
\end{abstract}

Keywords: Mikania glomerata, Asteraceae, coumarin, spectrophotometer, methylparaben, syrup.

\section{INTRODUÇÃO}

A Mikania glomerata Sprengel pertence a família Asteraceae, popularmente conhecida como "guaco", "cipó-caatinga", "guaco-cheiroso" e "erva-deserpente". É uma planta nativa da América Tropical e facilmente encontrada no Brasil sendo muito utilizada na medicina popular na forma de xaropes, para tratamento de afecções pulmonares devido ao seu efeito broncodilatador (Moura et al., 2001; Leite et al., 1993; Silva et al., 2006; Soares et al., 2006; Agra et al., 2008; Carvalho et al., 2008). Além de possuir outras atividades farmacológicas comprovadas experimentalmente como antioxidante (Vicentino \& Menezes, 2007), antidiarréica (Salgado et al., 2005) e antimicrobiana (Duarte et al., 2004). A importância dessa planta levou sua inclusão na Farmacopéia Brasileira I e IV (Brandão et al., 2006
$\& 2008)$.

A cumarina (1,2-benzopirona), é um dos principais constituintes da Mikania glomerata Sprengel (guaco) (Oliveira et al., 1985; Peregrino \& Leitão, 2005; Taleb-Contini et al., 2006). É definida como o marcador químico para controle de qualidade de formulações a base de guaco, conforme a Lista de registros simplificado de fitoterápicos da ANVISA, Resolução RE n ${ }^{\circ}$ 89, de 16 de março de 2004 (Anvisa, 2004).

As principais técnicas para doseamento de cumarinas em Mikania glomerata e seus derivados são por cromatografia líquida de alta eficiência (CLAE) e por cromatografia gasosa (Vilegas et al., 1997; Aboy et al., 2000; Celeghini et al., 2001,). Entretanto, as cumarinas apresentam espectro ultravioleta característico, devido à natureza e posição dos substituintes químicos, favorecendo sua identificação e o desenvolvimento 
de técnicas analíticas espectrofotométricas para o seu doseamento (Bruneton, 1991).

A forma farmacêutica mais utilizada para administração de extratos de Mikania glomerata Sprengel é o xarope contendo em sua composição constituintes como metil parabeno, sacarose, água, entre outros. Além da complexidade de constituintes encontrados no próprio extrato de Mikania glomerata Sprengel que podem interferir em um doseamento espectrofotométrico, pode-se destacar como potencial interferente o metil parabeno, que possui absorção máxima no comprimento de onda de $250 \mathrm{~nm}$, próximo ao pico de absorção da cumarina, $275 \mathrm{~nm}$ (Lozhkin \& Sakanyan, 2006), além de apresentar-se nos xaropes em concentração em torno de $1 \mathrm{mg} / \mathrm{mL}(0,1 \%)$, valor muito superior a concentração do marcador químico cumarina. O presente trabalho tem como objetivo desenvolver uma técnica por espectroscopia na região do ultravioleta para doseamento de cumarina em xaropes de Mikania glomerata Sprengel.

\section{MATERIAL E MÉTODOS}

As matérias-primas utilizadas foram, padrão secundário de 1,2-benzopirona (cumarina), de procedência Chromadex S.A. (USA) com um grau de pureza de 99,9\%, metil parabeno de grau farmacêutico (Galena Química Farmacêutica Ltda), clorofórmio (F. MAIA Indústria e Comércio Ltda), etano1 96 GL (Quimex Ltda), hexano (Vetec Química Fina LTDA), acetonitrila grau CLAE (Vetec Química Fina LTDA), e xarope de guaco contendo em sua formulação: açúcar (60\%), 0,1\% de metil parabeno, $10 \%$ tintura de Mikania glomerata Sprengel (Phitolabor Laboratório de Produtos Naturais Ltda) contendo $0,8 \mathrm{mg} / \mathrm{mL}$ de cumarina, água qsp. Os equipamentos utilizados para realização das análises foram, balança analítica (QUIMIS Q-500 L210C), espectrofotômetro de UV/visível Hitachi modelo U2010 e CLAE composto por bomba Shimadzu - LC 10AD; sistema de dados LC - Work Station Class LC -10; detector UV Shimadzu - SPD 10A e forno CTO10AS.

\section{Seleção do solvente para extração da cumarina}

Devido á complexidade da matriz optou-se por realizar a extração da cumarina da amostra. Para tanto, foi utilizado o método de Shake-flask para determinar o coeficiente de partição da mesma em dois solventes extratores, clorofórmio e hexano (Martin et al., 1993).

Foi preparada uma solução teste de $0,05 \mathrm{mg} /$ $\mathrm{mL}$ de cumarina em água, pré-solubilizada em etanol. Alíquota de $10 \mathrm{~mL}$ da mesma foi adicionada em um funil de separação juntamente com $10 \mathrm{~mL}$ de clorofórmio, manteve-se em leve agitação por 12 horas, separou-se a fase aquosa da fase clorofórmica. A concentração de cumarina na fase aquosa após partição, foi determinada por cromatografia líquida de alta eficiência (CLAE) utilizando fase móvel acetonitrila:água (40:60 v/v), fluxo $1,0 \mathrm{~mL} / \mathrm{min}$, temperatura de $30{ }^{\circ} \mathrm{C}$, detecção em $274 \mathrm{~nm}$, coluna Supelcosil $\mathrm{LC}_{18} 25 \mathrm{~cm} \times 4,6 \mathrm{~mm}, 5$ $\mu \mathrm{m}$, volume injetado de $20 \mu \mathrm{L}$ (Celeghini et al., 2001). O coeficiente de partição foi determinado dividindo a concentração de cumarina na fase clorofórmica pela concentração na fase aquosa. Este mesmo procedimento foi realizado com o solvente extrator hexano, sendo que todas as amostras foram analisadas em triplicata. O solvente selecionado foi aquele que apresentou maior coeficiente de partição.

\section{Seleção do comprimento de onda}

Foram obtidos espectros na região entre $400 \mathrm{e}$ 210 das seguintes soluções: A) cumarina á $0,008 \mathrm{mg} / \mathrm{mL}$; B) metil parabeno á $0,006 \mathrm{mg} / \mathrm{mL}$, ambos preparados utilizando o solvente selecionado para extração; C) diluição do xarope de guaco em meio aquoso com concentração final de cumarina á $0,008 \mathrm{mg} / \mathrm{mL}$; D) diluição obtida do seguinte procedimento extrativo: 10 $\mathrm{mL}$ do xarope de Mikania glomerata Sprengel contendo $0,08 \mathrm{mg} / \mathrm{mL}$ de cumarina (determinado por CLAE; Celeghini et al., 2001), foi adicionado a um funil de separação e em seguida foram extraídas três frações de $10 \mathrm{~mL}$ utilizando o solvente selecionado. Cada fração foi mantida em contato com o xarope por dez minutos. As três frações foram acondicionadas em balão volumétrico de $100 \mathrm{~mL}$ e completou-se o volume com o solvente selecionado. O comprimento de onda selecionado para o estudo foi aquele onde se observou sobreposição dos espectros da solução de cumarina $0,008 \mathrm{mg} / \mathrm{mL}$ e da solução obtida da diluição das frações extraídas do xarope de Mikania glomerata Sprengel.

\section{Curva analítica de cumarina}

Foram pesados $50 \mathrm{mg}$ de padrão de cumarina e transferidos para um balão volumétrico de $100 \mathrm{~mL}$, completando o volume com o solvente selecionado. A partir desta solução, foram preparadas amostras com concentrações de $0,002,0,005,0,008,0,02$ e $0,03 \mathrm{mg} /$ $\mathrm{mL}$. As absorbâncias foram determinadas em triplicata no comprimento de onda selecionado. Foram traçadas curvas analíticas de concentração versus absorbância para avaliar a linearidade das mesmas.

\section{Seletividade do processo de doseamento de cumarina na presença de metil parabeno}

Foram preparadas soluções contendo, $0,08 \mathrm{mg} /$ $\mathrm{mL}$ de cumarina mais $1 \mathrm{mg} / \mathrm{mL}$ de metil parabeno, e outra solução contendo $0,08 \mathrm{mg} / \mathrm{mL}$ de cumarina mais $1,5 \mathrm{mg} / \mathrm{mL}$ de metil parabeno. O teor de cumarina nas três soluções foi determinado utilizando o processo extrativo descrito no item "Seleção do comprimento de onda" com o solvente e comprimento de onda 
previamente selecionado, utilizando espectrofotômetro Hitachi U2010.

Comparação entre as técnicas CLAE e UV na quantificação de cumarina no xarope de Mikania glomerata

Amostra de $10 \mathrm{~mL}$ do xarope de Mikania glomerata Sprengel contendo $0,08 \mathrm{mg} / \mathrm{mL}$ de cumarina (determinado por CLAE conforme método descrito por Celeghini et al., 2001) foi adicionado a um funil de separação e em seguida foram extraídas três frações de $10 \mathrm{~mL}$ utilizando o solvente selecionado. Cada fração foi mantida em contato com o xarope por dez minutos. As três frações foram acondicionadas em balão volumétrico de $50 \mathrm{~mL}$ e completou-se o volume com o solvente selecionado. Em seguida foram determinadas as absorvâncias no espectrofotômetro Hitachi U2010, e através das curvas analíticas foi estimado as concentrações de cumarina nos xaropes. Para avaliar a precisão do procedimento as amostras foram analisadas em triplicata em três lotes de xarope. A média dos teores de cumarina determinados pelos métodos espectrofotométrico e por CLAE foram comparados por teste $t$ de Student e as precisões entre os métodos pelo teste F, utilizando software Origin 7.0.

\section{RESULTADOS E DISCUSSÃO}

\section{Seleção do solvente}

Os xaropes de Mikania glomerata Sprengel, além de cumarina possuem outros constituintes que podem interferir na análise espectroscópica, e por este motivo optou-se trabalhar com um processo extrativo da cumarina utilizando solventes como clorofórmio e hexano, visto que o logaritmo do coeficiente de partição octanol/água $(\log \mathrm{P})$ da cumarina é igual a 1,39 (Yourick \& Bronaugh, 1997). O coeficiente de partição determinado para cumarina em hexano e clorofórmio está apresentado na Tabela 1.

O clorofórmio apresentou capacidade de extração 36,4 vezes maior que o hexano. Por este motivo, optou-se por utilizá-lo para desenvolvimento da técnica.

\section{Seleção do comprimento de onda}

Para determinar o comprimento de onda de menor interferência do metil parabeno e dos outros constituintes do xarope sobre a cumarina, foram sobrepostos os espectros de soluções clorofórmicas de cumarina, metil parabeno, diluição do xarope de Mikania glomerata Sprengel antes do processo extrativo e diluição da fase clorofórmica extraída a partir do xarope, Figura 1.
O pico máximo de absorção da solução contendo apenas metil parabeno encontra-se em 250 nm (B), sendo que o espectro da solução com 0,008 $\mathrm{mg} / \mathrm{mL}$ de cumarina (A) apresentou dois picos de absorção, um em $275 \mathrm{~nm}$ e outro em aproximadamente $317 \mathrm{~nm}$. O espectro da diluição do xarope de Mikania glomerata Sprengel (C) contendo concentração idêntica de cumarina $0,008 \mathrm{mg} / \mathrm{mL}$ (determinada por CLAE cromatograma na Figura 2) à solução do espectro (A), demonstrou elevada interferência dos constituintes do xarope, impossibilitando uma análise direta entre 400 a $240 \mathrm{~nm}$. Entretanto, o espectro (D) referente as diluições das frações clorofórmicas extraída a partir

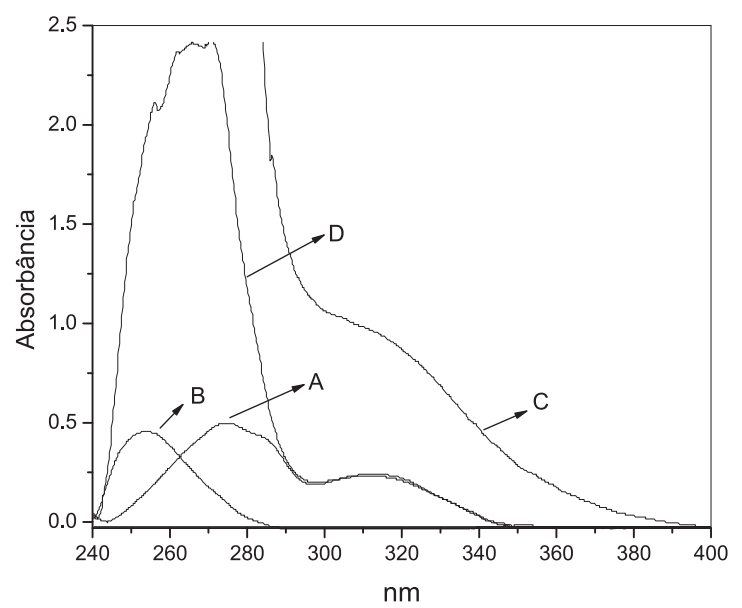

Figura 1. Sobreposição dos espectros obtidos entre 400 e 240 $\mathrm{nm}$ de: (A) cumarina $0,008 \mathrm{mg} / \mathrm{mL}$; (B) metil parabeno 0,006 $\mathrm{mg} / \mathrm{mL}$; (C) diluição do xarope de guaco em meio aquoso com concentração final de cumarina á $0,008 \mathrm{mg} / \mathrm{mL}$; D) diluição das frações clorofórmicas extraídas do xarope de Mikania glomerata Sprengel.

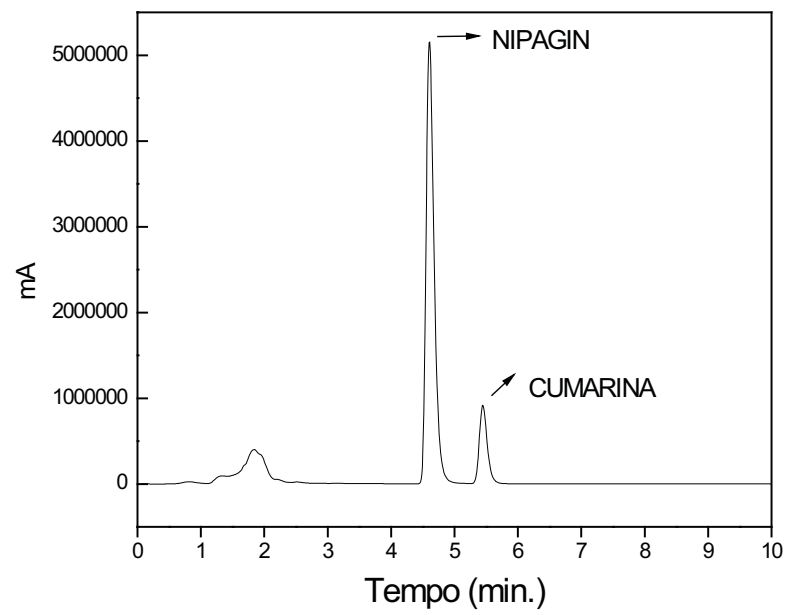

Figura 2. Cromatograma da diluição do xarope de Mikania glomerata Sprengel com concentração de cumarina á $0,008 \mathrm{mg} /$ $\mathrm{mL}$. 
do xarope de Mikania glomerata Sprengel apresentou sobreposição ao espectro da cumarina (A) entre 350 e $300 \mathrm{~nm}$, demonstrando que o processo extrativo foi eficiente para reduzir à níveis aceitáveis a concentração dos interferentes para o desenvolvimento da técnica. Baseado nestes resultados, o comprimento de onda selecionado foi de $320 \mathrm{~nm}$.

\section{Curva analítica e linearidade}

Na tabela 2 estão apresentados os valores de coeficiente de correlação, da intersecção da reta (a) e os valores da inclinação da reta (b) de duas curvas analíticas obtidas em $320 \mathrm{~nm}$ para cumarina em clorofórmio.

Os coeficientes de correlação citados na tabela 2, demonstraram que as curvas analíticas apresentaram relação linear entre a concentração e a absorbância, sendo que o critério mínimo aceitável, segundo RE $\mathrm{n}^{\circ}$ 29 de maio de 2003, é de 0,99 (Anvisa, 2003).

\section{Seletividade do processo de doseamento de cumarina na presença de metil parabeno}

O metil parabeno é utilizado geralmente a $0,1 \%$ em xaropes, concentração 12,5 vezes maior que a de cumarina encontrada no xarope teste $(0,08 \mathrm{mg} / \mathrm{mL})$,

Tabela 1. Coeficientes de partição da cumarina entre fase aquosa e as fases clorofórmica e hexânica.

\begin{tabular}{cccc}
\hline Solvente & $\begin{array}{c}\text { Concentração na fase orgânica } \\
(\mathrm{mg} / \mathrm{mL})\end{array}$ & $\begin{array}{c}\text { Concentração na } \\
\text { fase aquosa }(\mathrm{mg} / \mathrm{mL})\end{array}$ & $\mathrm{P}$ \\
\hline Hexano & 0,0275 & 0,0225 & 1,2222 \\
Clorofórmio & 0,0489 & 0,0011 & 44,4545 \\
\hline
\end{tabular}

P: partição

Tabela 2. Valores de coeficiente de correlação, intersecção da reta (a) e inclinação da reta (b) obtidos da regressão linear das curvas analíticas obtidas.

\begin{tabular}{ccccc}
\hline Análise & $\mathrm{R}$ & $\mathrm{R}^{2}$ & $\mathrm{a}$ & $\mathrm{b}$ \\
\hline $1^{\circ}$ & 0,99989 & 0,999780 & $4,69 \cdot 10^{-4}$ & 37,700 \\
$2^{\circ}$ & 0,99988 & 0,99976 & $5,32 \cdot 10^{-3}$ & 38,485 \\
\hline & Média \pm DP & & $2,900.10^{-3} \pm 0,002$ & $38,092 \pm 0,392$ \\
\hline
\end{tabular}

Tabela 3. Seletividade do processo de doseamento de cumarina na presença de metil parabeno.

\begin{tabular}{cccrr}
\hline $\begin{array}{c}\text { Concentração teórica de } \\
\text { Cumarina }(\mathrm{mg} / \mathrm{mL})\end{array}$ & $\begin{array}{c}\text { Concentração de metil } \\
\text { parabeno }(\mathrm{mg} / \mathrm{mL})\end{array}$ & $\begin{array}{c}\text { Concentração de cumarina } \\
\text { quantificada }(\mathrm{mg} / \mathrm{mL}) \pm \mathrm{DP}\end{array}$ & $t_{c}$ & $t_{t}$ \\
\hline 0,0800 & 0,0 & $0,079 \pm 7,100 \cdot 10^{-4}$ & & \\
0,0800 & 1,0 & $0,079 \pm 4,553 \cdot 10^{-4}$ & 0,302 & 9,925 \\
0,0800 & 1,5 & $0,078 \pm 7,953.10^{-4}$ & 0,406 & 9,925 \\
\hline
\end{tabular}

DP: desvio padrão $\mathrm{n}=3 ; \mathrm{p}<0,05 ; t_{c}$ : valor de $\mathrm{t}$ calculado; $t_{t}$ : valor de $\mathrm{t}$ tabelado.

Tabela 4. Concentração de cumarina detectada nos xaropes de Mikania glomerata sprengel por cromatografia líquida de alta eficiência (CLAE) e pela técnica espectrofotométrica (UV), e valores de teste $t$ de Student e teste F.

\begin{tabular}{|c|c|c|c|c|c|}
\hline Amostra & $\begin{array}{c}\text { Concentrações CLAE } \pm \text { DP } \\
(\mathrm{mg} / \mathrm{mL})\end{array}$ & $\begin{array}{c}\text { Concentrações UV } \pm \mathrm{DP} \\
(\mathrm{mg} / \mathrm{mL})\end{array}$ & $\begin{array}{l}\text { Média } \\
(\mathrm{CV} \%) \\
\end{array}$ & $t_{\mathrm{c}}$ & $F_{c}$ \\
\hline \multirow{3}{*}{1} & \multirow{3}{*}{$0,0730 \pm 0,0002$} & $0,0728 \pm 0,0002$ & \multirow{3}{*}{$\begin{array}{c}0,0729 \\
(1,10)\end{array}$} & \multirow{3}{*}{0,139} & \multirow{3}{*}{16,33} \\
\hline & & $0,0722 \pm 0,0001$ & & & \\
\hline & & $0,0738 \pm 0,0004$ & & & \\
\hline \multirow{3}{*}{2} & \multirow{3}{*}{$0,0740 \pm 0,0002$} & $0,0726 \pm 0,0002$ & \multirow{3}{*}{$\begin{array}{r}0,0735 \\
(1,30)\end{array}$} & \multirow{3}{*}{0,832} & \multirow{3}{*}{22,58} \\
\hline & & $0,0735 \pm 0,0001$ & & & \\
\hline & & $0,0745 \pm 0,0002$ & & & \\
\hline \multirow{3}{*}{3} & \multirow{3}{*}{$0,0760 \pm 0,0001$} & $0,0766 \pm 0,0002$ & \multirow{3}{*}{$\begin{array}{l}0,0762 \\
(0,52)\end{array}$} & \multirow{3}{*}{0,693} & \multirow{3}{*}{16,33} \\
\hline & & $0,0761 \pm 0,0001$ & & & \\
\hline & & $0,0758 \pm 0,0002$ & & & \\
\hline
\end{tabular}

$\mathrm{DP}=$ desvio padrão; $t=4,60$ para nível de significância de $0,01, \mathrm{~F}$ crítico $=21,20$ para nível de significância de 0,01 . 
além de apresentar máximo de absorbância em 250 $\mathrm{nm}$, fatores que o tornam um potencial interferente da técnica de doseamento.

Na tabela 3, estão representados os valores da concentração de cumarina determinados através das absorbâncias em $320 \mathrm{~nm}$ de amostras em soluções contendo diferentes concentrações de metil parabeno.

$O$ resultado do teste $t$ com $(\mathrm{p}<0,05)$ da comparação entre os teores de cumarina detectados nas amostras com ausência e presença de metil parabeno em concentrações 12,5 e 18,75 vezes maiores que as de cumarina nas soluções, demonstrou que esta substância não interfere significativamente no doseamento.

\section{Comparação entre as técnicas CLAE e UV na quantificação de cumarina no xarope de Mikania glomerata}

$\mathrm{Na}$ tabela 4, estão apresentados os valores das concentrações de três lotes de xarope de Mikania glomerata Sprengel com teor de cumarina determinado pela técnica cromatográfica descrita por Celeghini et al., (2001) e os valores das concentrações obtidas pelo doseamento espectrofotométrico em $320 \mathrm{~nm}$.

Através do teste $t$ de Student para um nível de significância de 0,01 , foi demonstrado não existir diferenças significativas entre as médias do teor de cumarina detectado pela técnica espectrofotométrica em $320 \mathrm{~nm}$ comparada a técnica por CLAE, indicativo de exatidão do método. Os coeficientes de variação para as quantificações de cada amostra analisado apresentaram valores inferiores a $1,30 \%$, demonstrando precisão na análise espectrofotométrica. Os valores calculados para o teste $\mathrm{F}$ demonstram que para a análise da amostra 1 e 3 não foram observados diferenças entre as precisões dos métodos, entretanto para amostra 2 ocorreu diferença significativa. Este comportamento é devido a baixa variação das quantificações realizadas por CLAE, enquanto que para o preparo das amostras para análise espectrofotométrica é necessário a extração da cumarina, fator que pode gerar maior desvio entre os resultados.

\section{CONCLUSÃO}

Os resultados demonstraram que a metodologia de doseamento da cumarina através de extração com clorofórmio e detecção por espectrofotometria em 320 $\mathrm{nm}$ apresenta-se como uma alternativa exata e precisa à CLAE para doseamento em xarope de Mikania glomerata Sprengel contendo como conservante metil parabeno.

\section{AGRADECIMENTOS}

Os autores agradecem a FAPESC, UNISUL e PHITOLABOR Laboratórios de Produtos Naturais, pelo suporte financeiro.

\section{REFERÊNCIAS}

Aboy AL, Ortega GG, Petrovick PR, Langeloh A, Bassani VL 2000. Desenvolvimento tecnológico de soluções extrativas de Mikania glomerata Sprengel (Asteraceae), guaco. Rev Bras Cienc Farm 36: 165172 .

Agra MF, Silva KN, Basílio IJLD, França PF, Barbosa-Filho JM 2008. Survey of medicinal plants used in the region Northeast of Brazil. Rev Bras Farmacogn 18: 472-508

Anvisa 2003. Agência Nacional de Vigilância Sanitária, Resolução RE Nº 899, de 29 de Maio de 2003. Diário Oficial da República Federativa do Brasil, Brasília DOU de 02/06/2003.

Anvisa 2004. Agência Nacional de Vigilância Sanitária. Resolução $\mathrm{n}^{\circ}$ 89, de 16 de março de 2004. Diário Oficial da República Federativa do Brasil, Brasília DOU de 18/03/2004.

Brandão MGL, Cosenza GP, Moreira RA, Monte-Mor RLM 2006. Medicinal plants and other botanical products from the Brazilian Official Pharmacopoeia. Rev Bras Farmacogn 16: 408-420.

Brandão MGL, Zanetti NNS, Oliveira GRR, Goulart LO, Monte-Mor RLM 2008. Other medicinal plants and botanical products from the first edition of the Brazilian Official Pharmacopoeia. Rev Bras Farmacogn 18: 127-134.

Bruneton J 1991. Elementos de fitoquimica y farmacognosia. Zaragoza: Acribia.

Carvalho ACB, Balbino EE, Maciel A, Perfeito JPS 2008. Situação do registro de medicamentos fitoterápicos no Brasil. Rev Bras Farmacogn 18: 314-319.

Celeghini RMS, Vilegas JHY, Lanças FM 2001. Extraction and quantitative HPLC analysis of coumarin in hidroalchoolic extracts of Mikania glomerata Sprengel (guaco). J Braz Chem Soc 12: 706-709.

Duarte MCT, Figueira GM, Pereira B, Magalhães PM, Delarmelina C 2004. Atividade antimicrobina de extratos hidroalcoólicos de espécies da coleção de plantas medicinais CPQBA/UNICAMP. Rev Bras Farmacogn 14 (Supl.1): 6-8.

Leite MGR, Souza CL, Silva MAM, Moreira LK, Matos FJA, Viana GSB 1993. Estudo Farmacológico comparado de Mikania glomerata Sprengel (gauco), Justicia pectoris Jacq (anador) e Torresea cearensis Fr. All (cumaru). Rev Bras Farm 74: 12-25.

Lozhkin AV, Sakanyan EI 2006. Natural coumarins: Methods of isolation and analysis. Pharm Chem J 40: 337346.

Martin A, Bustamante P, Chun AHC 1993. Physical pharmacy: physical chemical principles in the pharmaceutical sciences. Philadelphia: Lippincott Williams \& Wilkins.

Moura RS, Costa SS, Jansen JM, Silva CA, Lopes CS, Filho MB, Silva VN, Portela BN, Rubenich LMS, Araújo, RG, Carvalho LCRM 2001. Bronchodilator activity of Mikania glomerata Sprengel on human bronchi and guinea-pig trachea. J Pharm Pharmacol 54: 249256

Oliveira F, Akisue G, Akisue MK 1985. Parâmetros físicos e químicos e efeito antiedema dos extratos fluídos de guaco (Mikania glomerata Sprengel). An Farm Quim 
São Paulo 25: 50-54.

Peregrino CAF, Leitão SG 2005. Chromatographical profi les of fl uid extracts and tinctures obtained from Mikania glomerata Sprengel sterilized by gamma ray irradiation. Rev Bras Farmacogn 15: 237-242.

Salgado HRN, Roncari AFF, Moreira RRD 2005. Antidiarrhoeal effects of Mikania glomerata Spreng. (Asteraceae) leaf extract in mice. Rev Bras Farmacogn 15: $205-$ 208.

Silva MIG, Gondim APS, Nunes IFS, Sousa FCF 2006. Utilização de fitoterápicos nas unidades básicas de atenção à saúde da família no município de Maracanaú (CE). Rev Bras Farmacogn 16: 455-462.

Soares AKA, Carmo GC, Quental DP, Nascimento DF, Bezerra FAF, Moraes MO, Moraes MEA 2006. Avaliação da segurança clínica de um fitoterápico contendo Mikania glomerata, Grindelia robusta, Copaifera officinalis, Myroxylon toluifera, Nasturtium officinale, própolis e mel em voluntários saudáveis. Rev Bras Farmacogn 16: 447-454.

Taleb-Contini SH, Santos PA, Veneziani RCS, Pereira AMS, França SC, Lopes NP, Oliveira DCR 2006. Differences in secondary metabolites from leaf extracts of Mikania glomerata Sprengel obtained by micropropagation and cuttings. Rev Bras Farmacogn 16 (Supl.): 596-598.

Yourick JJ, Bronaugh RL 1997. Percutaneous absorption and metabolism of coumarin in human and rat skin. $J$ Appl Toxicol 17: 153-158.

Vicentino ARR, Menezes FS 2007. Atividade antioxidante de tinturas vegetais, vendidas em farmácias com manipulação e indicadas para diversos tipos de doenças pela metodologia do DPPH. Rev Bras Farmacogn 17: 384-387.

Vilegas JHY, Marchi E, Lanças FM 1997. Determination off coumarin and kaurenoic acd in Mikania glomerata (guaco) leaves by capillary gas chromatography. Phytochem Anal 8: 74-77. 